\title{
Brucellosis and rheumatic syndromes in Saudi Arabia
}

\author{
WALTER L. NORTON \\ From the Division of Rheumatology, Department of Medicine, King Faisal Specialist Hospital and Research \\ Centre, Riyadh 11211, Saudi Arabia
}

SUMMARY Seventy-three episodes of brucellosis in 55 patients are reported. Twenty-two had a systemic illness, 10 arthritis, 10 spondylitis, and four arthritis and spondylitis. Cultures were positive in $77 \%$ of cases where appropriate methods were used. A number of rheumatic syndromes may be mimicked by brucellosis and others may coexist with it. A high index of suspicion should prevail in the evaluation of patients from endemic areas.

Key words: infectious arthritis, spondylitis.

The practise of rheumatology in Saudi Arabia includes the same rheumatic syndromes as are encountered in the West, but their frequencies differ. Brucellosis is so prevalent that it must be considered in the differential diagnosis of many Saudi Arabian patients seen in the rheumatology clinic. Brucellosis at the King Faisal Specialist Hospital (KFSH) is reviewed. Brief case reports illustrate some of the clinical presentations encountered in rheumatology.

\section{Case reports}

\section{CASE 1}

A 72-year-old diabetic man was treated for culture positive brucellosis. He sought medical care abroad for recurrent discomfort and was thought to have polymyalgia rheumatica because of a raised erythrocyte sedimentation rate (ESR), morning stiffness, malaise, and myalgias. $20 \mathrm{mg}$ prednisone daily provided moderate symptomatic improvement.

On his return to Riyadh the ESR was $8 \mathrm{~mm} / \mathrm{h}$ and brucella titre $1 / 1280$. There was mild diffuse muscle tenderness. There was a mild increase in symptoms when corticosteroids were tapered. When blood culture became positive, he was treated with antibiotics for six weeks.

Four months later the same syndrome recurred. The ESR was $16 \mathrm{~mm} / \mathrm{h}$, brucella titre $1 / 640$, and blood culture was again positive for brucella.

CASE 2

A 60-year-old woman with azotaemia, ischaemic

Accepted for publication 7 June 1984.

Correspondence to $\mathrm{Dr} \mathrm{W}$. L. Norton. heart disease, hypertension, and diabetes complained of pain and swelling of the left knee for one weèk. She was afebrile. A large, tense knee effusion was aspirated and injected with corticosteroids. The fluid was negative for crystals, the ESR was 62 $\mathrm{mm} / \mathrm{h}$, rheumatoid factor, and a slide test and synovial fluid culture for brucella were negative. Two weeks after injection the knee was painless, and there was a small effusion.

Two months later she suffered fever, malaise, arthralgia, and recurrent knee swelling. The brucella titre was $1 / 320$. A blood culture was positive. With antibiotic treatment she became afebrile, and the arthritis and arthralgia subsided.

\section{CASE 3}

An 80-year-old man noted progressive symmetrical polyarthritis for one year. He had morning stiffness, malaise, weight loss, fever, and backache. He regularly drank unpasteurised camel, sheep, and goat milk. His temperature was $38^{\circ} \mathrm{C}$. There were large effusions of the shoulders, knees, and extensor tendon sheaths of the wrists. The ESR was $67 \mathrm{~mm} / \mathrm{h}$ and a rheumatoid factor test was negative. The brucella titre was $1 / 2560$. Granulomatous inflammation was found on synovial biopsy, and a synovial fluid culture was positive. Destructive lesions of vertebrae T11 and L1 were seen on $x$-ray. The bone scan is shown in Fig. 1.

Fever, effusions, and malaise resolved with treatment, but he relapsed several months later.

\section{CASE 4}

A 20-year-old male student complained of progressive pain and swelling of the knee for two months. 


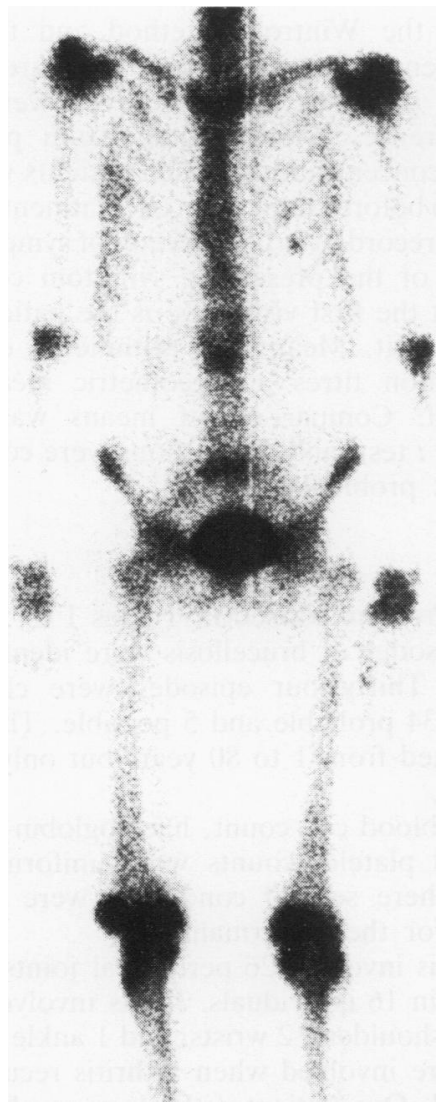

Fig. 1 Bone scan of case 3 showing symmetrical polyarthritis as well as spinal involvement.

He had sacrificed a lamb two months previously and drunk unboiled camel milk six months previously. There was soft tissue swelling and effusion of the right knee. The ESR was $40 \mathrm{~mm} / \mathrm{h}$. Microscopic haematuria was noted on two occasions. The brucella titre was $1 / 320$ and blood culture was positive.

With antibiotic treatment there was resolution of symptoms, arthritis, and haematuria. The ESR fell to $4 \mathrm{~mm} / \mathrm{h}$.

\section{CASE 5}

A 43-year-old man with back pain was found to have erosion of the anterior border of L3 (Fig. 2a) and a brucella titre of $1 / 400$. Three routine blood cultures held for only seven days were negative.

Two years after treatment he returned with recurrent severe low back pain. The brucella titre was 0 and the ESR was $6 \mathrm{~mm} / \mathrm{h}$. There was osteophyte formation at the site of previous erosion (Fig. 2b).

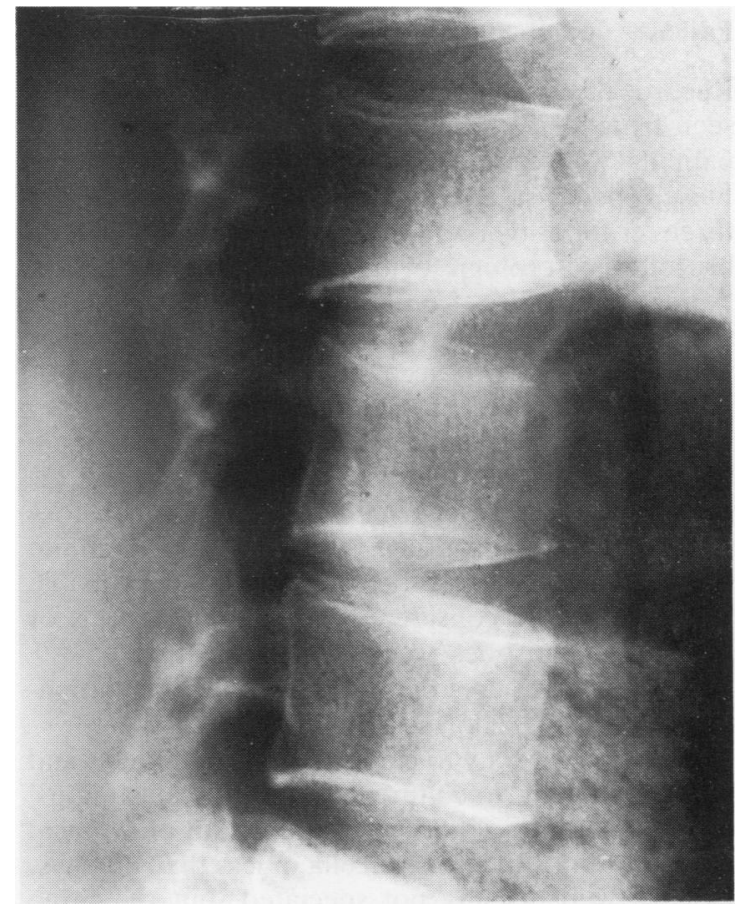

Fig. 2 A: Case 5 at time of initial infection. There is squaring and erosion of the anterior border of $L 3$.

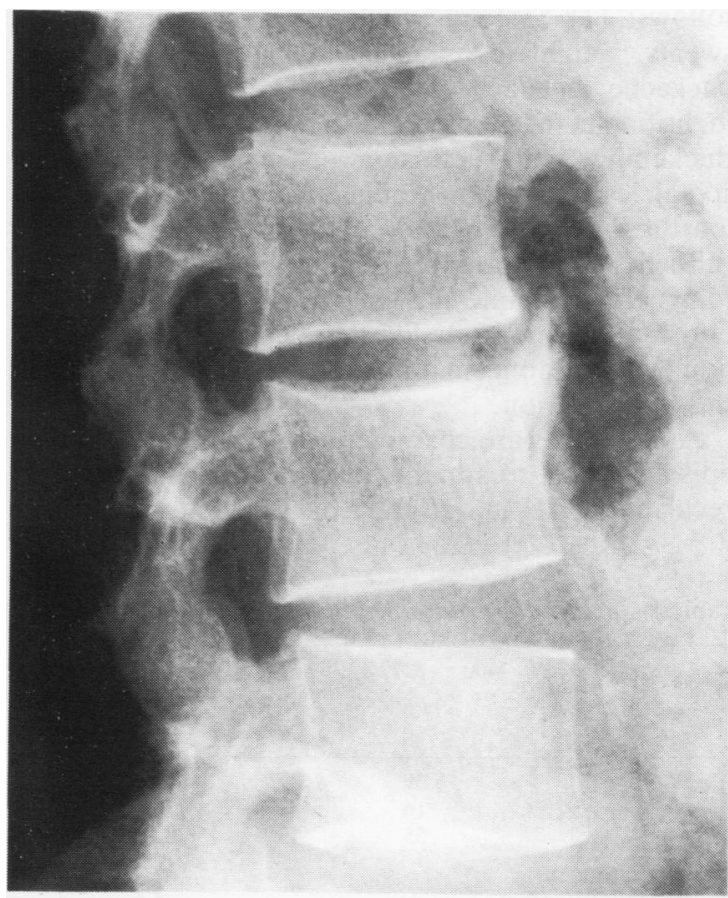

Fig. 2 B: Osteophyte has formed two years later. 


\section{Patients and methods}

Records of patients with the diagnosis of brucellosis seen from 1977 through 1984 were reviewed. Only patients whose diagnosis was established at King Faisal Specialist Hospital and who met one of the three criteria below were included. (1) Possible brucellosis: serologic evidence of brucellosis (titre $>1 / 160$ or a rise from 0 to $1 / 80$ or higher), a syndrome consistent with brucellosis and no other identified disease to account for the clinical condition, but who had an equivocal or unsatisfactory response to treatment or were lost to follow-up before response to treatment could be assessed. (2) Probable brucellosis: as in (1) but with a satisfactory response to appropriate therapy. (3) Definite brucellosis: culture positive. Episodes of probable brucellosis were considered definite if previous or subsequent episodes were culture positive.

Brucella titres are reported as the tube agglutination titre using abortus antigen.

Results refer only to cultures kept for 30 days and exclude short-term cultures not kept for 'fastidious' organisms, since only two cultures, both of synovial fluid, were positive for brucella in less than 30 days. Isolates were usually not speciated, but the three isolates identified during the time of this review were $B$. melitensis.

Episodes have been classified into clinical types as follows: (1) Systemic: symptoms including fever, sweats, weight loss, malaise, myalgias, arthralgia, backache, headache, nausea and vomiting without identifiable focal infection. (2) Arthritis: synovial thickening and/or effusion. (3) Spondylitis: focal spinal lesions demonstrable on $x$-ray. (4) Spondyloarthritis: fulfilling criteria for both (2) and (3). (5) Miscellaneous: focal infection not involving spine or joints, for example meningitis, abscess, or pleural effusion. Abnormal liver function, hepatosplenomegaly or abnormal liver biopsy were ignored in the classification.

Assessments are characterised according to initial episode, post-treatment period, and relapse or recurrence. About one-third of ESR determinations were by the Wintrobe method and the rest by Westergren. Results of both methods are pooled for statistical purposes. Globulin levels were taken as the difference between total serum protein and albumin concentrations. When patients were lost to follow-up before completion of treatment, follow-up time was recorded as 0 . Duration of symptoms is the duration of the presenting symptom complex recorded at the first visit. Age is the patient's age at the first visit. Means are arithmetic, except that agglutination titres are geometric means of the reciprocal. Comparison of means was done by Student's $t$ test, and proportions were compared by the exact probability test. ${ }^{1}$

\section{Results}

Results are summarised in Tables 1 to 5 . Seventythree episodes of brucellosis were identified in 55 patients. Thirty-four episodes were classified as definite, 34 probable and 5 possible. The patients' ages ranged from 1 to 80 years but only two were under 15.

White blood cell count, haemoglobin concentration, and platelet counts were uniformly normal except where second conditions were present to account for the abnormalities.

Arthritis involved 26 peripheral joints during 20 episodes in 16 individuals. Joints involved were 16 knees, 3 shoulders, 2 wrists, and 1 ankle. The same joints were involved when arthritis recurred in an individual. One patient with olecranon bursitis and pleural effusion is included in the miscellaneous group.

Synovial fluid analysis from eight joints had a mean cell count 17.5 (SD 13.7) $\times 10^{9} / 1$ (range 4.9 $\left.46 \times 10^{9} / l\right)$. Polymorphonuclear cells averaged $65 \%$ of the synovial fluid cells (range $23-91 \%$ ). Synovial fluid glucose in four specimens was 109, 104, 46, and $76 \mathrm{mg} / \mathrm{dl}$. $(6 \cdot 0,5 \cdot 8,2 \cdot 6$, and $4 \cdot 2 \mathrm{mmol} / \mathrm{l})$. Protein in three specimens was $5.5,5.0$ and $3.7 \mathrm{~g} / \mathrm{dl}(55,5$, and $37 \mathrm{~g} / \mathrm{l})$.

Spinal involvement at one or more levels occurred in 15 patients. The cervical spine was involved in

Table 1 Presenting syndromes and duration of symptoms at the initial attack

\begin{tabular}{|c|c|c|c|c|c|c|c|}
\hline & \multirow{2}{*}{$\begin{array}{l}\text { No. of } \\
\text { patients }\end{array}$} & \multirow{2}{*}{$\begin{array}{l}\text { Mean } \\
\text { age }\end{array}$} & \multicolumn{5}{|c|}{ Initial presenting syndrome } \\
\hline & & & Systemic & Arthritis & Spondylitis & Spondylo-arthritis & Misc. \\
\hline $\begin{array}{l}\text { Male } \\
\text { Female } \\
\text { Total }\end{array}$ & $\begin{array}{l}39 \\
16 \\
55\end{array}$ & $\begin{array}{l}47 \\
41 \\
45\end{array}$ & $\begin{array}{l}11 \\
11 \\
22\end{array}$ & $\begin{array}{r}8 \\
2 \\
10\end{array}$ & $\begin{array}{r}10 \\
0 \\
10\end{array}$ & $\begin{array}{l}4 \\
0 \\
4\end{array}$ & $\begin{array}{l}6 \\
3 \\
9\end{array}$ \\
\hline $\begin{array}{l}\text { Mean duration of } \\
\text { symptoms (months) }\end{array}$ & & & $5 \cdot 6$ & $6 \cdot 8$ & $4 \cdot 7$ & $9 \cdot 8$ & $4 \cdot 5$ \\
\hline
\end{tabular}


Table 2 Recurrences and follow-up (FU) times

\begin{tabular}{|c|c|c|c|c|c|c|c|}
\hline \multirow[t]{2}{*}{$\begin{array}{l}\text { Initial presenting } \\
\text { syndrome }\end{array}$} & \multirow[t]{2}{*}{$\begin{array}{l}\text { Total number } \\
\text { of patients }\end{array}$} & \multicolumn{3}{|c|}{$\begin{array}{l}\text { Number of patients } \\
\text { with relapses }\end{array}$} & \multirow{2}{*}{$\begin{array}{l}\text { Mean } F U \\
\text { patients with } \\
\text { relapse (months) }\end{array}$} & \multirow{2}{*}{$\begin{array}{l}\text { Mean FU } \\
\text { patients without } \\
\text { relapse (months }\end{array}$} & \multirow{2}{*}{$\begin{array}{l}\text { Mean } F U \\
\text { all patients } \\
\text { (months) }\end{array}$} \\
\hline & & Total & $M$ & $F$ & & & \\
\hline Systemic & 22 & 9 & 6 & 3 & $14 \cdot 4$ & $5 \cdot 4$ & $9 \cdot 1$ \\
\hline Arthritis & 10 & 5 & 5 & 0 & $16 \cdot 5$ & $2 \cdot 2$ & 7.9 \\
\hline Spondylitis & 10 & 3 & 3 & 0 & $28 \cdot 0$ & $9 \cdot 25$ & $13 \cdot 0$ \\
\hline Spondylo-arthritis & 4 & 1 & 1 & 0 & $13 \cdot 0$ & $12 \cdot 0$ & $12 \cdot 3$ \\
\hline Miscellaneous & 9 & 0 & 0 & 0 & - & $4 \cdot 1$ & $4 \cdot 1$ \\
\hline Total or mean of & 55 & 18 & 15 & 3 & $17 \cdot 3 \pm 11$ & $6 \cdot 1 \pm 9$ & $9 \cdot 0$ \\
\hline
\end{tabular}

Table 3 Results of brucella cultures

\begin{tabular}{|c|c|c|c|c|c|c|c|}
\hline \multirow[t]{2}{*}{ Initial syndrome } & \multicolumn{5}{|c|}{ Initial presenting syndrome } & \multirow[t]{2}{*}{ All recurrences } & \multirow[t]{2}{*}{ Line total } \\
\hline & Systemic & Arthritis & Spondylitis & Spondylo-arthritis & Misc. & & \\
\hline \multicolumn{8}{|l|}{ Source: } \\
\hline Blood & $9 / 9$ & $4 / 6$ & - & $0 / 1$ & $1 / 2$ & $2 / 2$ & $16 / 20$ \\
\hline Synovial fluid & - & $3 / 4$ & - & $1 / 1$ & - & $4 / 4$ & $8 / 9$ \\
\hline Other & - & $1 / 1$ & $1 / 1$ & - & $1 / 4$ & - & $3 / 6$ \\
\hline Total & $9 / 9$ & $8 / 11$ & $1 / 1$ & $1 / 2$ & $2 / 6$ & $6 / 6$ & $27 / 35$ \\
\hline
\end{tabular}

$-=$ None done.

Numerator $=$ number of cultures positive.

Denominator $=$ number of episodes cultured properly.

Multiple cultures done on the same source at the same time are considered to be a single culture, since there were no instances of discrepancies in results from multiple cultures. Therefore the figures represent a 'single culture' per source per episode.

Table 4 Fever, weight loss, and pain in initial and recurrent attacks

\begin{tabular}{llr}
\hline & $\begin{array}{l}\text { Initial attacks (\%) } \\
n=55\end{array}$ & $\begin{array}{l}\text { Recurrence (\%) } \\
n=17\end{array}$ \\
\hline Fever & $44(80)$ & $9(53)$ \\
Weight loss & $14(25)$ & $2(12)$ \\
Arthralgia & $26(47)$ & $12(71)$ \\
Backache & $25(45)$ & $1(6)$ \\
Headache & $12(22)$ & $1(6)$ \\
\hline
\end{tabular}

Table 5 Reciprocal agglutinin titres, ESR, and globulin levels in initial and recurrent episodes and in asymptomatic post-treatment periods

\begin{tabular}{|c|c|c|c|c|c|c|}
\hline & \multicolumn{2}{|c|}{ Initial episode } & \multicolumn{2}{|c|}{ Recurrent episodes } & \multicolumn{2}{|c|}{ Post-treatment } \\
\hline & $(n)$ & mean $\pm S D$ & $(n)$ & mean $\pm S D$ & (n) & mean $\pm S D$ \\
\hline \multirow[t]{2}{*}{ Brucella agglutinin titre } & (53) & $765 \pm 1 \cdot 35^{*}$ & (17) & $11141 \pm 1 \cdot 14^{*}$ & $\begin{array}{r}(31) \\
\mathrm{p}<0.001\end{array}$ & $145 \pm 2 \cdot 34^{*}$ \\
\hline & & L & & $\mathrm{p}<0.001$ & & \rfloor \\
\hline ESR & (43) & $44 \pm 19$ & (10) & $\begin{array}{c}19 \pm 19 \\
p<0.001\end{array}$ & (7) & $6 \pm 6$ \\
\hline Globulin & $(48)$ & $3 \cdot 8$ & $(9)$ & $3 \cdot 6$ & (4) & $2 \cdot 8$ \\
\hline
\end{tabular}

${ }^{*}$ Geometric mean \pm SD $1 n$. 
four, thoracic spine in five, and lumbar spine in eight patients. Spinal involvement and orthopaedic experience with arthritis will be reported in greater detail elsewhere by R. Lifeso.

\section{Discussion}

In this series $60 \%$ of patients had focal complications (Table 1) as opposed to less than $10 \%$ in abattoir acquired disease. ${ }^{2}$ This high proportion may reflect not only brucella species differences but the relatively long duration of symptoms before treatment in the present series-5.4 months compared with less than one month in Buchanan and colleagues' series. ${ }^{2}$

The average age of 45 years is nearly double that in Rajaee's series ${ }^{3}$ and is best explained by a systematic underrepresentation of the paediatric age group in this survey. Eleven of 22 patients with the systemic syndrome were female, whereas only two of the 24 remaining patients were female $(p=0 \cdot 01)$.

The recurrence rate was more than $50 \%$ if only patients followed for six months or more are included. This may be due partly to statistical artefact, since the reason for longer follow-up may have been recurrence of symptoms. It is likely that the true recurrence rate lies between the overall rate of $30 \%$ (Table 2) and the $55 \%$ of those with prolonged follow-up. Recurrences were probably due both to a high rate of reinfection and true recurrence after inadequate treatment, though Buchanan et al. classify all recurrences within three years as relapses. ${ }^{2}$ Comparable information on recurrence and duration of follow-up is not available from other published series.

The ESR, agglutinin, and globulin levels fell significantly with treatment. During recurrent attacks the ESR was lower than during the initial attack, hepatosplenomegaly was less prominent, and there were fewer symptoms (Tables 4 and 5). This probably reflects more rapid diagnosis in recurrent cases.

The brucella isolation rate of $77 \%$ compares well with the $72 \%$ reported in an abattoir epidemic ${ }^{2}$ and is much higher than in other clinical series. These results refer only to results of cultures submitted specifically for brucella. Many other cultures in the same patients were negative when brucellosis was not specified on the culture request-for example Case 5. Blood cultures were usually requested by the departments of internal medicine and rarely by surgery, and synovial fluid cultures for brucella were most frequently requested by the rheumatology department, at times by orthopaedics, and rarely by internal medicine.

In most cases of the systemic type features such as abnormal liver function tests (about $60 \%$ ), hepatosplenomegaly (about $50 \%$ ), or prolonged fever suggested the correct diagnosis, but liver, spleen, or lymph node enlargement was relatively infrequent in arthritis and spondylitis patients $(<10 \%)$.

One of 12 latex tests for rheumatoid factor was positive $(1 / 40)$, and two of seven ANA tests were positive $(1 / 120)$. These three positive results were obtained from culture positive patients.

Bone scans confirmed the results of conventional radiological examination, but their usefulness in identifying otherwise occult foci of destructive disease was not demonstrated. One gallium scan was performed, and it was negative in a patient with systemic disease.

No predisposing illnesses were noted. The incidence of diabetes was not excessive for the population as a whole, and only two of eight diabetics had a recurrence.

This series does not conform with the traditional division into 'acute' and 'chronic' brucellosis, ${ }^{4}$ and the utility of such a classification is questionable.

Late exuberant osteophyte formation after infection was not prominent, but case 5 (Fig. 2) is a good example of this phenomenon.

It has been suggested that reactive arthritis may be caused by brucellosis. ${ }^{56}$ The results of this series suggest that probably all clinical brucellosis is due to active infection, since culture results are excellent if appropriate isolation techniques are used, and since patients responded to antibiotic treatment as would be expected in active infection.

Though spondylitis is often severely destructive, peripheral joint arthritis is usually more benign and responds well to antibiotic treatment. Regular synovial fluid aspiration, as is recommended in pyogenic infection, ${ }^{7}$ was not performed in any of these patients, and good results were noted in all who could be followed up after treatment.

$A$ raised ESR, hyperglobulinaemia, and nonspecific symptoms brought many rheumatic syndromes into the differential diagnosis even when joints were not involved. Two patients were thought to have polymyalgia rheumatica. The clinical pictures of fibrositis, systemic lupus erythematosus, rheumatoid arthritis, and Pott's disease were mimicked. Brucellosis also coexisted with chondrocalcinosis and degenerative joint disease.

Two patients were treated for polymyalgia rheumatica with corticosteroids, with resulting fall in ESR. Two patients received intra-articular corticosteroids. All four had temporary symptomatic improvement.

Brucellosis in the West is largely confined to meat handlers, ${ }^{2} 8$ but in developing countries milk and meat products are widely contaminated at the 
consumer level, so that the disease occurs over a much wider segment of society. Therefore brucellosis must be considered in the differential diagnosis of many rheumatological syndromes when the patient is from an endemic area such as the Middle East, south Asia, ${ }^{3}$ and Africa. ${ }^{4} 910$

The cooperation of Dr Edward Harder and Dr Robert Lifeso is greatly appreciated. Dr Theodore Bailey in the Clinical Laboratory. Department of Pathology, supervised the culture techniques during most of the time covered in this report. Appreciation is also expressed to Mrs Yvonne Lock and Miss Vilma Contreras, Department of Medicine.

\section{References}

1 Swinscow T D V. Statistics at square one. London: British Medical Association. 1981.
2 Buchanan T M, Faber L C, Feldman R A. Brucellosis in the United States 1968-1972. Medicine 1974; 53: 403-13.

3 Rajaee A. Brucella arthritis. Iranian J Med Sci 1980; 11: 45-59.

4 Thimm B. Brucellosis in Uganda. Bull Epizoot Dis Afr 1972; 20: $43-56$.

5 Hodinko L. Gömör B, Meretéy K. et al. HLA B27-associated spondylarthritis in chronic brucellosis. Lancet 1978; i: 499.

6 Alarcon G S, Boganegra T S, Gottinzo E, et al. Reactive arthritis associated with brucellosis: HLA studies. J Rheumatol $1981 ; 8: 621-5$.

7 Goldenberg D L. Brandt K D, Cohen A S, et al. Treatment of septic arthritis: comparison of needle aspiration and surgery as initial modes of joint drainage. Arthritis Rheum 1975; 18: 83-90.

8 Bigler W J, Hoff G L. Hemmert W H, et al. Trends of brucellosis in Florida: An epidemiologic review. Am J Epidemiol 1977; 105: 245-51.

9 Sacks N. Van Rensburg A J. Clinical aspects of chronic brucellosis. S Afr Med J 1976; 50: 725-8.

10 Osman L J A. Human brucellosis in Kenya. Trop Geogr Med 1976; $28: 45-53$. 